\title{
Trichoscopy of scalp dysesthesia
}

\author{
Adriana Rakowska, Małgorzata Olszewska, Lidia Rudnicka \\ Department of Dermatology, Medical University of Warsaw, Warsaw, Poland
}

Adv Dermatol Allergol 2017; XXXIV (3): 245-247

DOI: https://doi.org/10.5114/pdia.2017.67148

\begin{abstract}
Introduction: Scalp dysesthesia is characterized by localized pruritus, burning sensations or even pain.

Aim: To describe characteristic trichoscopic features of scalp dysesthesia.

Material and methods: The study was a retrospective analysis (2010-2016) of 9 patients (8 females and 1 male; median age: 52 years; range: 38-66 years). A total of 61 trichoscopic images were analyzed by two independent evaluators.

Results: In all cases, oval or round areas covered with short hairs: broom hairs (77\%; 7/9), block hairs (88\%; 8/9), short hairs with trichorrhexis nodosa (78\%; 7/9) were found. In 4 of 9 cases these areas were circumscribed by brownish linear discoloration. Background features included: areas lacking hair shafts (44\%; 4/9) covered by wavy-shaped scaling, whitish areas with prominent perifollicular yellow discoloration and cloud vessels arranged around follicular openings and around empty follicles (33\%, 3/9); focal brownish discoloration with darker wavy lines (78\%; 7/9).

Conclusions: Trichoscopy can be helpful in establishing the diagnosis of scalp dysesthesia - broom hairs, block hairs and short hairs with trichorrhexis nodosa covering the alopecia patch, uniform in length, can be considered as characteristic for this condition.
\end{abstract}

Key words: scalp dysesthesia, alopecia, dermoscopy, trichoscopy, pruritus.

\section{Introduction}

Scalp dysesthesia was first described in 1998 by Hoss and Segal. It is characterized by localized pruritus, burning sensations or even pain [1]. The pathogenesis of this condition is poorly understood and has not been determined. The pain and pruritus may be related to the chronic tension placed on the occipitofrontalis muscle and scalp aponeurosis secondary to the underlying cervical spine disease [2].

There are only few publications about this condition $[2,3]$.

Aim

Trichoscopy (hair and scalp dermoscopy) is a rapid inoffice technique, which has become a standard procedure in differential diagnosis of hair loss [4-11]. There is only one case report addressing trichoscopy features in scalp dysesthesia [12]. The aim of this study was to assess characteristic trichoscopic features of scalp dysesthesia.

\section{Material and methods}

The study was a retrospective analysis (2010-2016). The trichoscopic database of 4532 records and patient records were searched to identify patients having been seen with scalp dysesthesia. Nine patients were identified (8 females/1 male; average age: 52 years, range: 4466 years; average disease duration: 16 months, range: 5-36 months). All of them had a single patch of focal alopecia $(1-3 \mathrm{~cm})$ with pruritus and burning. Most of these lesions were located in the vertex area of the scalp (7 of 9), one in the occipital area and one in the parietal area.

Trichoscopy was performed in each case (as a standard procedure in diagnosing hair loss). All pictures (total 61 images) were evaluated by two authors for the presence of specific abnormalities in the hair shaft structure and skin surface.

\section{Results}

In all cases oval or round areas covered with short hairs were found and in 4 of 9 cases they were circumscribed by brownish linear discoloration (Figures 1, 2). Short hairs covering the entire alopecia patch were quite uniform in length and were composed of broom hairs (short hairs with longitudinal splitting of hair shaft from distal ends to the follicular opening; also known as trichoptilosis) in 7 of 9 patients (78\%), block hairs (short hairs

Address for correspondence: Adriana Rakowska MD, PhD, Department of Dermatology, Medical University of Warsaw, 82 a Koszykowa St, 00-005 Warsaw, Poland, phone/fax: +48 2250213 24, e-mail: adriana.rakowska@gmail.com Received: 28.09.2016, accepted: 28.11.2016. 
with horizontal distal end) in 8 of 9 patients (89\%) and trichorrhexis nodosa in 7 of 9 patients (78\%) (Figures 2-5).

Background features included areas lacking hair shafts (44\%; 4/9) (Figure 2) covered by wavy-shaped scaling (wavy shape was visible because of "dirty" discoloration of scale margins; Figure 2); whitish areas with prominent perifollicular yellow discoloration and cloud

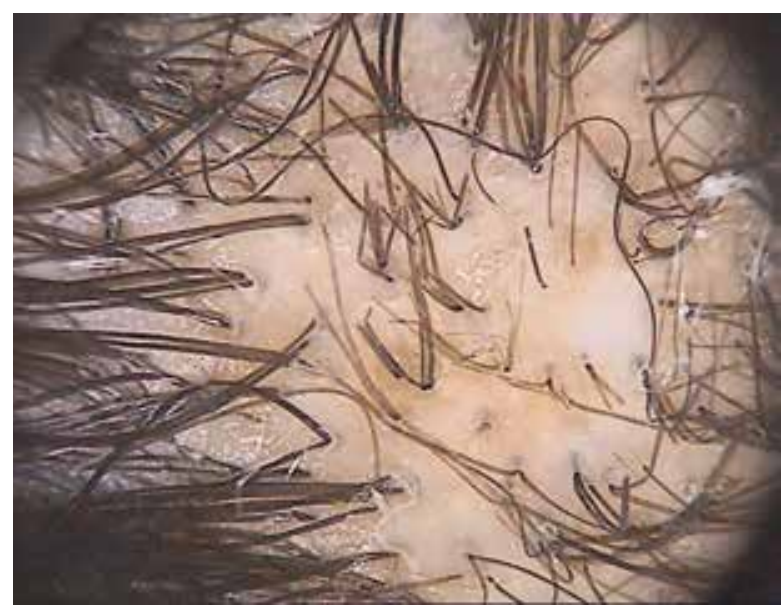

Figure 1. Trichoscopy of scalp dysesthesia. In the centra part, a round area covered with short hairs can be observed. Notice brownish discoloration of the background (20-fold magnification)

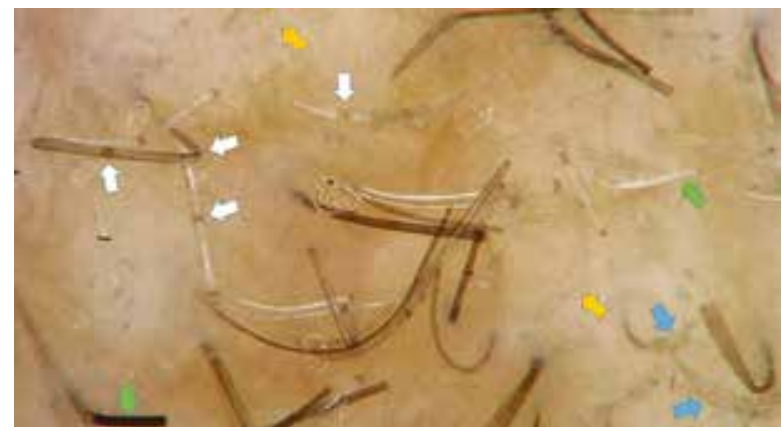

Figure 3. Trichoscopy of scalp dysesthesia. Brownish discoloration with darker wavy lines (yellow arrows) can be seen. Multiple short hairs can be observed: broom hairs (blue arrows), block hairs (green arrows) and trichorrhexis nodosa (white arrows) (70-fold magnification)

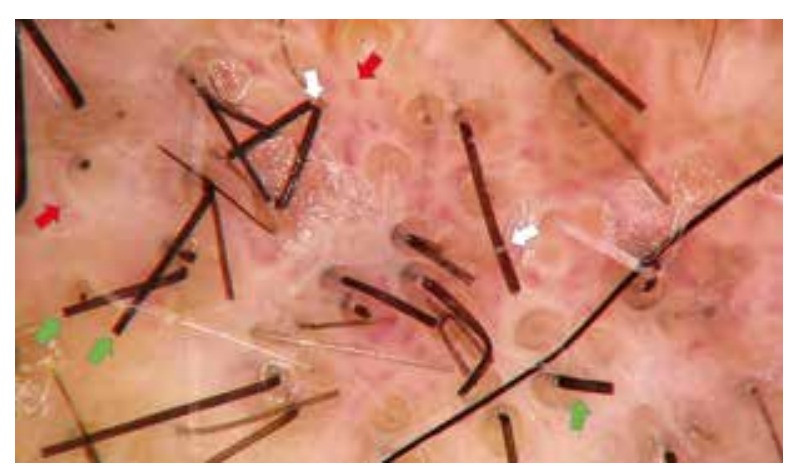

vessels arranged around follicular openings and around empty follicles (33\%; 3/9) (Figure 5); focal brownish discoloration with darker wavy lines (78\%; 7/9) (Figure 3). Round yellow structures reflecting pustules were found in 2 of 9 patients (22\%) (Figure 2). Some yellow dots (empty follicular openings filled with sebum) were also found $(33 \% ; 3 / 9)$.

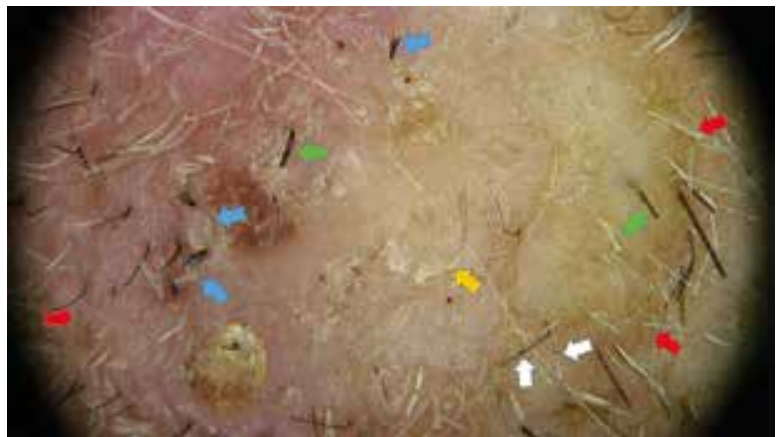

Figure 2. Trichoscopy of scalp dysesthesia. The alopecia patch is circumscribed by brownish linear discoloration (red arrows). An area lacking hair shafts covered by wavyshaped scaling (wavy shape can be visible because of "dirty" discoloration of scale margins) can be seen (yellow arrows). Multiple short hairs can be observed: broom hairs (blue arrows), block hairs (green arrows) and trichorrhexis nodosa (white arrow) (20-fold magnification)

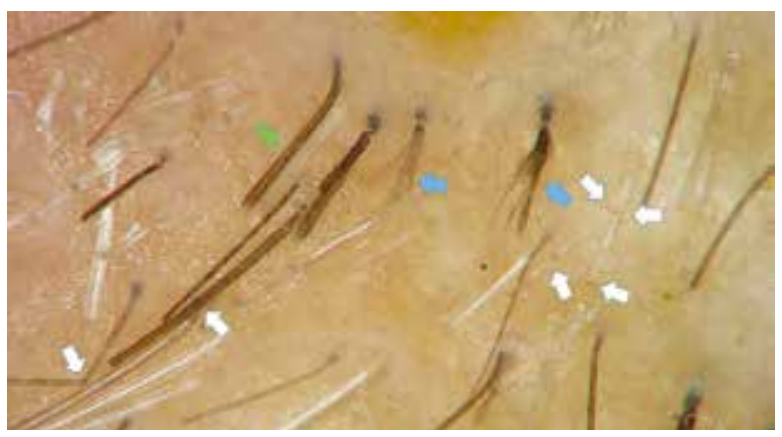

Figure 4. Trichoscopy of scalp dysesthesia. Trichorrhexis nodosa (white arrows); pay attention to the left side of the picture - two hair shafts in one follicular unit have nodosities at the same level (this confirmed the mechanical couse of these abnormalities). Broom hairs (blue arrows), and brownish background discoloration can be noticed (70fold magnifications)

Figure 5. Trichoscopy of scalp dysesthesia, presents with the whitish area with prominent perifollicular yellow discoloration and cloud vessels (red arrows) arranged around follicular openings and around empty follicles. Short hairs: block hairs (green arrows) and trichorrhexis nodosa (white arrow) (70-fold magnification) 


\section{Discussion}

There is only one description of trichoscopy in scalp dysesthesia (one case) [12]. In this paper the author suggests that trichoptilosis is one of the most characteristic finding of this disorder. In our study (although limited because of a small number of patients as the disease is rare) we found more features regarding hair shafts and background. All lesions were covered by short hairs more or less uniform in length. In higher magnifications (70x) they occurred to be broom hairs, block hairs and short hairs with trichorhexis nodosa. They are caused by a mechanical injury and it is surprising that typical findings of trichotillomania (irregularly broken hairs, flame hairs, coiled hairs, hook hairs, tulip hairs) [13] were not found. The explanation of this may be that hairs in the small area were rubbed (not pulled out) with the same force. There are no reports concerning trichoscopy of trichoteiromania. Based on our own experience, broom hairs and trichorrhexis nodosa can be also observed in this condition, but less regularly distributed in patches of scratching alopecia. The length of those short hairs is more variable.

Short hairs with trichorhexis nodoxa have to be distinguished from Morse code-like hairs seen in tinea capitis [14]. In each case the mycological examination has to be performed.

Background changes were seen in our study mostly as skin brownish discoloration with wavy darker lines and corresponded to hypertrophy of the epidermis caused by chronic scratching.

\section{Conclusions}

A single patch of alopecia in the vertex area with pruritus and pain has to be considered as scalp dysesthesia. Trichoscopy can be helpful in establishing the diagnosis broom hairs, block hairs and short hairs with trichorrhexis nodosa covering the alopecia patch, uniform in length, can be considered as characteristic for scalp dysesthesia.

\section{Conflict of interest}

The authors declare no conflict of interest.

\section{References}

1. Hoss D, Segal S. Scalp dysesthesia. Arch Dermatol 1998; 134: 327-30.

2. Thornsberry LA, English JC $3^{\text {rd }}$. Scalp dysesthesia related to cervical spine disease. JAMA Dermatol 2013; 149: 200-3.

3. Sarifakioglu E, Onur O. Women with scalp dysesthesia treated with pregabalin. Int J Dermatol 2013; 52: 1417-8.

4. Kowalska-Oledzka E, Slowinska M, Rakowska A, et al. Sensitivity and specificity of the trichoscopy. Indian I Dermatol Venereol Leprol 2012; 78: 636-7.

5. Rakowska A. Trichoscopy (hair and scalp videodermoscopy) in the healthy female. Method standardization and norms for measurable parameters. I Dermatol Case Rep 2009; 3: 14-9.

6. Rakowska A, Slowinska M, Kowalska-Oledzka E, et al. Trichoscopy of cicatricial alopecia. J Drugs Dermatol 2012; 11: 753-8.

7. Rakowska A, Zadurska M, Czuwara J, et al. Trichoscopy findings in loose anagen hair syndrome: rectangular granular structures and solitary yellow dots. J Dermatol Case Rep 2015; 9: 1-5.

8. Neri I, Starace M, Patrizi A, Balestri R. Corkscrew hair: a trichoscopy marker of tinea capitis in an adult white patient. JAMA Dermatol 2013; 149: 990-1.

9. Rudnicka L, Rakowska A, Kurzeja M, Olszewska M. Hair shafts in trichoscopy: clues for diagnosis of hair and scalp diseases. Dermatol Clin 2013; 31: 695-708.

10. Tosti A. Practice gaps: trichoscopy in clinical care: comment on "corkscrew hair". Arch Dermatol 2011; 147: 356.

11. Torres F, Tosti A, Misciali C, Lorenzi S. Trichoscopy as a clue to the diagnosis of scalp sarcoidosis. Int J Dermatol 2011; 50: 358-61.

12. Leroux MB. Importance of the trichoscopy in scalp dysesthesia. Our Dermatol Online 2013; 4: 501-2.

13. Rakowska A, Slowinska M, Olszewska M, Rudnicka L. New trichoscopy findings in trichotillomania: flame hairs, V-sign, hook hairs, hair powder, tulip hairs. Acta Derm Venereol 2014; 94: 303-6.

14. Rakowska A, Zadurska M, Czuwara J, et al. Trichoscopy of focal alopecia in children - new trichoscopic findings: hair bulbs arranged radially along hair-bearing margins in aplasia cutis congenita. Skin Appendage Disord 2016; 2: 1-6. 